\title{
Características clínicas y epidemiológicas de pacientes adultos con diagnóstico de tuberculosis extrapulmonar en el Hospital Hipólito Unanue de Tacna durante los años 2016-2018
}

Clinical and epidemiological characteristics of adult patients diagnosed with extrapulmonary tuberculosis at Hipolito Unanue Hospital in Tacna during the period 2016-2018

Caraterísticas clínicas e epidemiológicas de pacientes adultos com diagnóstico de tuberculose extrapulmonar no Hospital Hipólito Unanue de Tacna durante os anos 2016-2018

Astrid Milagros Mamani Loza ${ }^{1 a}$

Edwin Cecilio Condori Vargas ${ }^{2 b}$

Nayareth Nancy Mamani Loza ${ }^{10}$

\section{Resumen}

Objetivo: Conocer las características clínicas y epidemiológicas de pacientes adultos con diagnóstico de tuberculosis extrapulmonar en el Hospital Hipólito Unanue de Tacna en el periodo 2016-2018. Material y métodos: Estudio observacional, descriptivo. Se incluyó a todos los pacientes mayores de 14 años con diagnóstico de tuberculosis extrapulmonar, los datos se obtuvieron de las historias clínicas, fueron procesados en el programa estadístico SPSS v24, y presentados a través de tablas con frecuencias absolutas y relativas. Resultados: De 1247 pacientes con diagnóstico de tuberculosis, la frecuencia de tuberculosis extrapulmonar fue $9,62 \%$ (120 casos), de ellos, el 60,8 \% fue hospitalizado. Los pacientes se caracterizaron principalmente por ser de sexo masculino $(64,2 \%)$, de 20 a 29 años (25,8 \%), con estudios secundarios $(75 \%)$, procedentes de Tacna (74,2 \%). El 68,33\% tuvo el antecedente de padecer condiciones no modificables y el $25 \%$ tenía condiciones modificables. El $65,83 \%$ de los casos tenía comprometido el sistema respiratorio; el 14,17\%, el sistema enteroperitoneal; el 13,33\%, el sistema nervioso central; el 9,16\%, el sistema linfático; y el 8,33\%, el sistema tegumentario. El 12,5\% presentó tuberculosis multisistémica. El 56,96 \% de pacientes presentó fiebre y el 34,18\%, pérdida del peso, hiporexia y tos con expectoración. Conclusión: La pleura fue la localización más frecuente de tuberculosis extrapulmonar, el 18,3\% presentó tuberculosis pulmonar como condición no modificable, el 6,67\% VIH/SIDAy el $5 \%$ tuvo diabetes mellitus, asma y anemia.

Palabras clave: tuberculosis, tuberculosis extrapulmonar, epidemiología, signos, síntomas

\footnotetext{
${ }^{1}$ Universidad Privada de Tacna. Tacna, Perú

${ }^{2}$ Hospital Hipólito Unanue. Tacna, Perú

${ }^{a}$ Médico cirujano

${ }^{\mathrm{b}}$ Médico neumólogo

${ }^{\circ}$ Estudiante de Medicina Humana
} 


\begin{abstract}
Objective: To know the clinical and epidemiological characteristics of adult patients diagnosed with extrapulmonary tuberculosis at the Hipolito Unanue Hospital in Tacna during the period 2016-2018. Material and methods: Observational, descriptive study. All patients older than 14 year-old with a diagnosis of extrapulmonary tuberculosis were included, data were obtained from clinical records, were processed in the SPSS v24 statistical program, and presented through tables with absolute and relative frequencies. Results: Of 1247 patients diagnosed with tuberculosis, the frequency of extrapulmonary tuberculosis was $9.62 \%$ (120 cases), of which $60.8 \%$ were hospitalized. The patients were characterized mainly by being male $(64.2 \%)$, from 20 to 29 year-old (35.8 \%), with secondary studies (75\%), from Tacna (74.2\%). $68.33 \%$ had a history of non-modifiable conditions and $25 \%$ had modifiable conditions. $65.83 \%$ of cases involved the respiratory system; $14.17 \%$ involved the enteroperitoneal system; $13.33 \%$ involved the central nervous system; $9.16 \%$ involved the lymphatic system; and $8.33 \%$ involved the tegumentary system. $12.5 \%$ had multisystem tuberculosis. $56.96 \%$ of patients had fever and $34.18 \%$, weight loss, hyporexia and coughing with expectoration. Conclusion: The pleura was the most frequent location of extrapulmonary tuberculosis, $18.3 \%$ presented pulmonary tuberculosis as a non-modifiable condition, $6.67 \%$ HIVIAIDS and $5 \%$ had diabetes mellitus, asthma and anemia.
\end{abstract}

Keywords: tuberculosis, extrapulmonary tuberculosis, epidemiology, signs, symptoms

\title{
Resumo
}

Objetivo: Conhecer as características clínicas e epidemiológicas de pacientes adultos com diagnóstico de tuberculose extrapulmonar no Hospital Hipólito Unanue de Tacna no período 2016-2018. Material e métodos: Estudo observacional, descritivo. Todos os pacientes com mais de 14 anos com diagnóstico de tuberculose extrapulmonar foram incluídos, os dados foram obtidos a partir de registros clínicos, foram processados no programa estatístico SPSS V24, e apresentados através de tabelas com freqüências absolutas e relativas. Resultados: De 1247 pacientes com diagnóstico de tuberculose, a frequência de tuberculose extrapulmonar foi 9,62 \% (120 casos), dos quais 60,8 \% foram hospitalizados. Os pacientes caracterizaram-se principalmente por serem do sexo masculino (64,2\%), de 20 a 29 anos (35,8 \%), com estudos secundários (75\%), procedentes de Tacna (74,2\%). 68,33\% tinham antecedentes de condições inalteradas e $25 \%$ tinham condições modificáveis. $65,83 \%$ dos casos tinham comprometido o sistema respiratório, $14,17 \%$ o sistema enteroperitoneal, $13,33 \%$ o sistema nervoso central, 9,16 \% o sistema linfático e $8,33 \%$ o sistema tegumentário. $12,5 \%$ de doentes com tuberculose multisistémica. $56,96 \%$ dos pacientes apresentaram febre e $34,18 \%$, perda de peso, hiporexia e tosse com expectoração. Conclusão: A pleura foi a localização mais frequente de tuberculose extrapulmonar, 18,3\% apresentaram tuberculose pulmonar como condição não modificável, 6,67 \% HIVISIDA e 5 \% tiveram diabetes mellitus, asma e anemia.

Palavras-chave: tuberculose, tuberculose extrapulmonar, epidemiologia, sinais, sintomas

\section{Introducción}

La tuberculosis es una patología infectocontagiosa cuyo agente causal es el Mycobacterium tuberculosis, afecta a toda la población en el mundo, siendo un problema latente en salud pública de mucha importancia, ya que puede llevarnos a la muerte. ${ }^{1}$ Según el reporte de la Organización Mundial de la Salud (OMS) del año 2018, la tuberculosis pulmonar es considerada la primera causa de muerte debido a un agente infeccioso, causando 1,3 millones de muertes cada año. ${ }^{2}$

En América Latina, en el año 2017, fallecieron cerca de 18 mil personas; en el Perú, la tuberculosis es considerada una enfermedad endémica, ocupando el segundo lugar con el mayor número de casos en la región América Latina y Caribe. ${ }^{3}$ Existen muchos órganos 
afectados por esta bacteria, si bien se conoce que del $80 \%$ de personas el foco es pulmonar, el $20 \%$ son casos extrapulmonares. ${ }^{4}$ En el Perú no hay reportes detallados de esta presentación de tuberculosis, solo hacen referencia a que existe un $20 \%$ de presentaciones extrapulmonares. ${ }^{5}$

Hoy en día, la tuberculosis y sus variantes están tomando cada vez una mayor relevancia como objeto de estudio, debido a que presentan cuadros inespecíficos y se ha visto que los casos van en aumento, asimismo, el conocimiento que se tiene es limitado y el diagnóstico muchas veces tardío. ${ }^{6}$ Es por ello que, el objetivo del presente trabajo fue conocer las características clínicas y epidemiológicas en pacientes adultos con diagnóstico de tuberculosis extrapulmonar del Hospital Hipólito Unanue de Tacna, y con ello, poder contribuir al conocimiento de esta patología, para realizar actividades preventivas y promocionales, mejorar el diagnóstico y dar tratamiento oportuno.

\section{Material y métodos}

Estudio observacional, descriptivo. La población del estudio comprendió todos los pacientes mayores de 14 años de ambos sexos, con diagnóstico de tuberculosis extrapulmonar atendidos en el Hospital Hipólito Unanue en la ciudad de Tacna durante el periodo 2016 a 2018, seleccionando las historias clínicas de aquellos pacientes con información completa de las variables principales de estudio.
Se solicitó los permisos correspondientes para acceder a la sección de archivos de la Unidad de Estadística del Hospital Hipólito Unanue de Tacna, se revisó las historias clínicas de pacientes que reunieron los criterios de selección y los datos fueron recogidos en una ficha pre elaborada, siendo posteriormente ingresados a una hoja electrónica, previamente codificados, conformando una base de datos.

Los datos fueron procesados y analizados utilizando el programa estadístico SPSS v24. Se presentan mediante tablas descriptivas con frecuencias absolutas $y$ relativas.

\section{Resultados}

Durante los años 2016 a 2018, en el Hospital Hipólito Unanue de Tacna se atendieron 1247 pacientes con tuberculosis, de ellos, 120 casos fueron tuberculosis con compromiso extrapulmonar, los cuales fueron objeto del análisis en el presente estudio.

La mayoría de los pacientes con tuberculosis extrapulmonar fueron hospitalizados (73 casos). El grupo etario más afectado fue de 20 a 29 años $(25,8 \%)$ y de los que requirieron hospitalización el mayor porcentaje fue el grupo etario mayor de 60 años. Respecto al sexo, 64,2 \% fueron mujeres; en cuanto al grado de instrucción, el $75 \%$ de casos tenían estudios secundarios; y en cuanto al lugar de procedencia el $74,2 \%$ fueron del distrito de Tacna (Tabla 1). 


\section{Tabla 1}

Características sociodemográficas en pacientes con tuberculosis extrapulmonar en el Hospital Hipólito Unanue de Tacna, 2016-2018

\begin{tabular}{|c|c|c|c|c|c|c|c|c|}
\hline & & \multicolumn{6}{|c|}{ Hospitalización } & \multirow{3}{*}{$p$} \\
\hline & & \multicolumn{2}{|c|}{ Sí } & \multicolumn{2}{|c|}{ No } & \multicolumn{2}{|c|}{ Total } & \\
\hline & & $\mathrm{N}$ & $\%$ & $\mathrm{n}$ & $\%$ & $\mathrm{n}$ & $\%$ & \\
\hline \multirow{7}{*}{ Edad } & $<20$ años & 16 & 21,9 & 8 & 17 & 24 & 20 & \multirow{8}{*}{0.222} \\
\hline & 20 a 29 años & 16 & 21,9 & 15 & 31,9 & 31 & 25,8 & \\
\hline & 30 a 39 años & 13 & 17,8 & 5 & 10,6 & 18 & 15 & \\
\hline & 40 a 49 años & 4 & 5,5 & 6 & 12,8 & 10 & 8,3 & \\
\hline & 50 a 59 años & 5 & 6,8 & 6 & 12,8 & 11 & 9,2 & \\
\hline & 60 a más & 19 & 26 & 7 & 14,9 & 26 & 21,7 & \\
\hline & Total & 73 & 100 & 47 & 100 & 120 & 100 & \\
\hline \multirow{3}{*}{ Sexo } & Femenino & 26 & 35,6 & 17 & 36,2 & 43 & 35,8 & \\
\hline & Masculino & 47 & 64,4 & 30 & 63,8 & 77 & 64,2 & \multirow[t]{2}{*}{0,951} \\
\hline & Total & 73 & 100 & 47 & 100 & 120 & 100 & \\
\hline \multirow{5}{*}{$\begin{array}{l}\text { Grado de } \\
\text { instrucción }\end{array}$} & Analfabeto/a & 5 & 6,8 & 0 & 0 & 5 & 4,2 & \multirow{5}{*}{0} \\
\hline & Primaria & 18 & 24,7 & 1 & 2,1 & 19 & 15,8 & \\
\hline & Secundaria & 44 & 60,3 & 46 & 97,9 & 90 & 75 & \\
\hline & Superior & 6 & 8,2 & 0 & 0 & 6 & 5 & \\
\hline & Total & 73 & 100 & 47 & 100 & 120 & 100 & \\
\hline \multirow{8}{*}{ Procedencia } & Tacna & 53 & 72,6 & 36 & 76,6 & 89 & 74,2 & \multirow{8}{*}{0,030} \\
\hline & Locumba & 5 & 6,8 & 5 & 10,6 & 10 & 8,3 & \\
\hline & La Yarada & 2 & 2,7 & 4 & 8,5 & 6 & 5 & \\
\hline & Penal Pocollay & 5 & 6,8 & 0 & 0 & 5 & 4,2 & \\
\hline & Puno & 6 & 8,2 & 0 & 0 & 6 & 5 & \\
\hline & Lima & 0 & 0 & 2 & 4,3 & 2 & 1,7 & \\
\hline & Sama & 2 & 2,7 & 0 & 0 & 2 & 1,7 & \\
\hline & Total & 73 & 100 & 47 & 100 & 120 & 100 & \\
\hline
\end{tabular}

En la tabla 2, se puede observar que las principales condiciones no modificables en pacientes con tuberculosis extrapulmonar fueron TBC pulmonar y VIH/SIDA; y en cuanto a las condiciones modificables fueron obesidad y desnutrición (Tabla 3). 


\section{Tabla 2}

Principales condiciones no modificables en pacientes con tuberculosis extrapulmonar en el Hospital Hipólito Unanue de Tacna, 2016-2018

\begin{tabular}{lcc}
\hline \multicolumn{1}{c}{ Condiciones no modificables } & $\mathrm{N}^{\circ}$ & $\%$ \\
\hline TBC pulmonar & 22 & 18,3 \\
VIH/SIDA & 8 & 6,67 \\
Asma & 7 & 5,83 \\
Anemia & 7 & 5,83 \\
Diabetes mellitus & 7 & 5,83 \\
ITU & 4 & 3,33 \\
Arritmia cardiaca & 4 & 3,33 \\
Neoplasia & 4 & 3,33 \\
Desnutrición crónica & 3 & 2,50 \\
Gastritis & 3 & 2,50 \\
Hipertensión arterial & 2 & 1,67 \\
Psoriasis & 2 & 1,67 \\
Hidrocefalia & 2 & 1,67 \\
Hidrocele & 2 & 1,67 \\
Insuficiencia cardíaca & 2 & 1,67 \\
Hepatitis & 2 & 1,67 \\
Sífilis & 1 & 0,83 \\
Enfermedad renal crónica & 1 & 0,83 \\
Hipertrofia de próstata & 1 & 0,83 \\
\hline
\end{tabular}

\section{Tabla 3}

Principales condiciones modificables en pacientes con tuberculosis extrapulmonar en el Hospital Hipólito Unanue de Tacna, 2016-2018

\begin{tabular}{lcr}
\hline \multicolumn{1}{c}{ Condiciones modificables } & $\mathrm{N}^{\circ}$ & $\%$ \\
\hline Obesidad & 9 & 7,50 \\
Desnutrición & 7 & 5,83 \\
Alcoholismo & 6 & 5 \\
Tabaquismo & 5 & 4,17 \\
Medicamentos y/o drogas & 4 & 3,33 \\
Adicción a drogas & 1 & 0,83 \\
Cambio de sexo & 1 & 0,83 \\
\hline
\end{tabular}

En la tabla 4 se presenta las principales ubicaciones de la tuberculosis extrapulmonar, observándose que el $65,83 \%$ se ubicaba en el sistema respiratorio; $14,17 \%$, en el sistema enteroperitoneal; y $13,33 \%$, en el sistema nervioso central. Presentó compromiso del sistema linfático $9,16 \%$, seguido por el sistema tegumentario con un $8,33 \%$. Cabe destacar que en la tabla 4 no se presentan los totales puesto que un paciente pudo tener más de una localización. 


\section{Tabla 4}

Localización por sistemas en pacientes con tuberculosis extrapulmonar en el Hospital Hipólito Unanue de Tacna, 2016-2018

\begin{tabular}{lcc}
\hline Localización de TB extrapulmonar & $\mathrm{N}^{\circ}$ & $\%$ \\
\hline Sistema respiratorio (Tuberculosis pleural) & 79 & 65,83 \\
Sistema enteroperitoneal & 17 & 14,17 \\
Sistema nervioso central & 16 & 13,33 \\
Multisistémico & 15 & 12,50 \\
Sistema linfático & 11 & 9,16 \\
Sistema tegumentario & 10 & 8,33 \\
Sistema genito-urinario & 9 & 7,50 \\
Sistema cardiovascular & 2 & 3,33 \\
\hline
\end{tabular}

En la tabla 5 observamos que el $56,96 \%$ de los pacientes presentó fiebre, seguido del $34,18 \%$, que presentó pérdida del peso, hiporexia y tos expectorante, respectivamente.

\section{Tabla 5}

Signos y síntomas de la localización principal de tuberculosis extrapulmonar: sistema respiratorio (tuberculosis pleural)

\begin{tabular}{lcc}
\hline \multicolumn{1}{c}{ Signos y síntomas } & $\mathrm{N}^{\circ}$ & $\%$ \\
\hline Fiebre & 45 & 56,96 \\
Pérdida de peso & 27 & 34,18 \\
Hiporexia & 27 & 34,18 \\
Tos con expectoración & 27 & 34,18 \\
Dolor torácico & 23 & 29,11 \\
Dolor pleurítico & 18 & 22,78 \\
Diaforesis & 8 & 10,13 \\
Malestar general & 7 & 8,86 \\
Sensación de alza térmica & 7 & 8,86 \\
Dificultad respiratoria & 7 & 8,86 \\
Náuseas y vómitos & 5 & 6,33 \\
Sudoración nocturna & 4 & 5,06 \\
Prurito & 4 & 5,06 \\
Dolor abdominal y dorsal & 3 & 3,80 \\
Lipotimia & 3 & 3,80 \\
Somnolencia & 3 & 3,80 \\
Diarrea & 2 & 2,53 \\
Baja de peso & 2 & 2,53 \\
Agitación/fatiga/cansancio & 2 & 2,53 \\
Escalofríos & 2 & 2,53 \\
Hinchazón de piernas & 2 & 2,53 \\
Cefalea & 1 & 1,27 \\
\hline
\end{tabular}




\section{Discusión}

Los dos tipos de manifestación clínica de la tuberculosis (TB) son la TB pulmonar (PTB) y la TB extrapulmonar (EPTB). El primero es el más común. EPTB se refiere a la TB que involucra órganos que no son pulmones (pleura, ganglios linfáticos, abdomen, tracto genitourinario, piel, articulaciones y huesos, o meninges). ${ }^{7}$ Ramírez et al. refieren que alrededor del $10-50 \%$ de los pacientes con EPTB tienen afectación pulmonar concomitante. Por lo tanto, todos los casos sospechosos de EPTB deben ser evaluados para determinar si el caso es infeccioso y ayudar con el diagnóstico. ${ }^{8}$ Yeon Lee refiere que el diagnóstico tardío de las formas extrapulmonares es frecuente y conlleva un aumento de la morbilidad y mortalidad. Los síntomas y signos pueden ser relativamente vagos y a veces se produce con exámenes laboratoriales y radiográficos negativos. ${ }^{9}$

En esta investigación se estudiaron los pacientes de tuberculosis extrapulmonar, quienes representaron por criterios de inclusión y exclusión, el 9,62 \% de 1247 pacientes con diagnóstico de tuberculosis. Dicho valor podría considerarse como frecuencia de periodo de los 3 años. Del grupo sujeto de investigación, el $64,2 \%$ era de sexo masculino y el $35,8 \%$ de sexo femenino. El $25,8 \%$ tenía entre 20 a 29 años y el $21,7 \%$ tenía de 60 años a más. El $75 \%$ contaba con estudios secundarios, el $74,2 \%$ procedía de Tacna. Un 4,2 \% procedían del penal de Pocollay. Brenes et al. ${ }^{10}$ encontraron que la edad promedio de los pacientes diagnosticados fue de 48 años y la relación de casos según sexo fue 2:1, predominando el sexo masculino. Por otra parte, Peñata et al. ${ }^{11}$ encontraron en su estudio que la mayoría de la población fueron hombres y la edad promedio fue de 42 años. Distribuciones diferentes comparadas a los resultados de la presente investigación, donde la frecuencia de casos se vio en menores de 30 años, principalmente.

Según localización, los hallazgos revelaron que el $65,83 \%$ fue en el sistema respiratorio, seguido del $14,17 \%$ en el sistema enteroperitoneal y un $13,33 \%$ en el sistema nervioso central. Se pudo observar que un $9,16 \%$ tenía comprometido el sistema linfático seguido en el quinto lugar por el sistema tegumentario con un $8,33 \%$. Brenes et al. ${ }^{10}$ encontraron que la localización más frecuente fue pleural (35\%), continuando con la ganglionar (28\%), meníngea (12\%), intestinal, ósea, peritoneal y otras. Por otra parte, Gomes de Barros et al. ${ }^{12}$ encontraron que la localización más frecuente fue pleural en un $(42,7 \%)$, seguida de la ganglionar $(40,1 \%)$ y la ósea (3,6 \%). Méndez et al. ${ }^{13}$ comentaron que los pacientes con tuberculosis extrapulmonar, requirieron más atención médica y hospitalización, y según su localización fueron de origen meníngeo, pleural y osteoarticular. Por otro lado, Chaves et al. ${ }^{14}$ publicaron un estudio donde las principales localizaciones fueron: pleural, pericárdica, peritoneal y meníngea. Pérez-Guzmán et al. ${ }^{15}$ encontraron que el sistema más comprometido en tuberculosis extrapulmonar fue el linfático.

Se estudió también las principales condiciones no modificables y modificables presentes, y se observó que el $18,3 \%$ tenía el antecedente de tuberculosis pulmonar seguido de un $6,7 \%$ con coinfección VIH/SIDA. En distribución proporcional similar, un 5,83\% presentaba asma, anemia y diabetes mellitus. Respecto a las condiciones modificables estuvo presente la obesidad, con un 7,5\% seguido de un $5,83 \%$ con desnutrición y un $5 \%$ alcoholismo.

Brenes et al. ${ }^{10}$ encontraron que el VIH fue el factor de riesgo más asociado a esta patología seguida de la diabetes mellitus, desnutrición, alcoholismo, neoplasias e insuficiencia renal crónica. Méndez et al. ${ }^{13}$ comentaron que la desnutrición fue el factor de riesgo que más se asoció con la presentación de tuberculosis; concluyendo que el mejor abordaje, tanto social, cultural y económico frente al comportamiento de la tuberculosis, es primordial y necesario para controlar esta enfermedad. Peñata et al. ${ }^{11}$ encontraron que la comorbilidad más frecuente fue el $\mathrm{VIH}$, seguida de la diabetes mellitus. Pérez-Guzmán et al. ${ }^{15}$ refieren que sus pacientes tenían antecedentes de diabetes mellitus, seguido de la obesidad, 
además, la mayoría de los pacientes eran jóvenes. En el presente estudio, la presentación principal fue en el sistema respiratorio, donde los signos y síntomas fueron de un $56,96 \%$ fiebre, seguido de un $34,18 \%$, distribuido de forma similar, entre pérdida del peso, hiporexia y tos con expectoración. Sandrino et al. ${ }^{16}$ afirmaron que, dado que a pesar de que la tuberculosis es una enfermedad curable aún no es un problema resuelto, por ello, recalcan la importancia de sospechar y mantener vigilancia de no solo la tuberculosis pulmonar, sino que también compromete otros órganos y sistemas. Coincidimos con su apreciación de que se necesita reforzar aún más las investigaciones que involucren características medio ambientales, nivel socioeconómico y estratos sociales, así como incursionar en el campo de la medicina ocupacional.

\section{Referencias}

1. Muñoz del Carpio-Toia $A$, Sánchez $H$, Verguez de López C, Sotomayor MA, Dávila LL, Sorokin P. Tuberculosis en América Latina y el Caribe: reflexiones desde la bioética. Pers. Bioét. 2018; 22(2):331-357.

2. World Health Organization (WHO) [Internet]. Global tuberculosis report 2018. D i s p o n i b I e e $n$ : https://www.who.int/tb/publications/global _report/en/.

3. Ministerio de Salud (MINSA) [Internet]. Boletín Epidemiológico del Perú. Volumen 27 -Semana Epidemiológica 07. 2018. D i s p o i b le e n : http://www.dge.gob.pe/portal/docs/vigilanc ia/boletines/2018/07.pdf

4. Valiente LR, Reto CP, Prieto OP, Cerna KD. Tuberculosis abdominal en niños y adolescentes. Un desafío diagnóstico. Rev Gastroenterol Perú. 9 de junio de 2017; 35(4):318-22.

5. Ministerio de Salud (MINSA) [Internet].Análisis de la situación epidemiológica de la tuberculosis en el Perú 2015. Disponible en: http://www.dge.gob.pe/portal/index.php?o ption=com_content $\&$ view $=$ article\&id=599: a $\mathrm{s} \quad \overline{\mathrm{i}} \mathrm{s} \quad \mathrm{c}$ t b c 2015\&catid=20:institucional\&ltemid=204

6. Villamizar JPV, Álvarez MFS, Sepúlveda JS, González ST, Méndez YR. Tuberculosis intestinal, un reto diagnóstico: a propósito de un caso. Horiz Méd Lima [Internet]. 30 de junio de 2016 [citado 10 de febrero de 2019]; 16(2). D is pon ible e n : http://www.horizontemedico.u smp.edu.pe/ind ex.php/ horizonte $\mathrm{med} / \mathrm{articl}$ e/view/432

7. Ministerio de Salud (MINSA) [Internet] Norma técnica de Salud para la atención integral de las personas afectadas por tuberculosis. 2013. Disponible en: http://www.tuberculosis.minsa.gob.pe/port aldpctb/recursos/20180308083418.pdf

8. Ramírez-Lapausa M, Menéndez-Saldaña A, Noguerado-Asensio A. Tuberculosis extrapulmonar, una revisión. Rev Esp Sanid Penit. Madrid, España. 2015; 17(1):3-11.

9. Lee JY. Diagnosis and Treatment of Extrapulmonary Tuberculosis. Tuberc Respir Dis. abril de 2015; 78(2):47-55.

10. Brenes MYM, Marchena MP. Epidemiología de la tuberculosis extrapulmonar en el Hospital Rafael Ángel Calderón Guardia: 2009-2013. Rev Médica Univ Costa Rica. 2014; 8(1):39-48.

11. Peñata $A$, Salazar R, Castaño, $T$, Bustamante J, Ospina S. Diagnóstico molecular de tuberculosis extrapulmonar y sensibilidad a rifampicina con un método automatizado en tiempo real. Biomédica Colombia. 2016; 36(1):78-89

12. Gomes de Barros P, Lima MP, Carla da Silva T, Luiz ES, Figueiredo F. Perfil Epidemiológico dos casos de Tuberculosis Extrapulmonar em un municipio de el estado de Paraíba, 2001-2010. Cad. saúde colet. Brasil; 22(4):343-350.

13. Méndez Fanidño $Y$, Caicedo Ochoa E, Urrutia Gómez J, Cortés Motta H, Ávila 
Espitia N, Álvarez Gallego G. Comparison of the Socioeconomic Factors and Diagnostic Test between Pulmonary Tuberculosis (PTB) and Extrapulmonary Tuberculosis (ETB) in Boyacá (Colombia), in 2015. Univ Médica. 19 de octubre de 2018; 59(4):1-8.

14. Chaves W, Buitrago JF, Dueñas A, Bejarano JC. Acerca de la tuberculosis extrapulmonar. Repert Med Cir. Colombia. 2017; 26(2):90-7.
15. Pérez-Guzmán $\mathrm{C}$, Vargas $\mathrm{MH}$, ArellanoMacías $M$ del $R$, Hernández-Cobos $S$, García-Ituarte AZ, Serna-Vela FJ. Clinical and epidemiological features of extrapulmonary tuberculosis in a high incidence region. Salud Pública México. abril de 2014; 56:189-96.

16. Sandrino-Sánchez $M$, Martínez-Muñoz $M$, Wong-Díaz L. Tuberculosis extrapulmonar. Presentación de un caso Medisur. Cuba. 2015; 13(3):442-447.

\section{Correspondencia:}

cecmax80@hotmail.com
Fecha de recepción: 12/08/2020

Fecha de aceptación: 18/01/2021 